\title{
Efficient contraction of 1-loop N-point tensor integrals
}

\author{
Jochem Fleischer* \\ Fakultät für Physik, Universität Bielefeld, Universitätsstr. 25, 33615 Bielefeld, Germany \\ E-mail: Fleischeraphysik.uni-bielefeld.de
}

\author{
Janusz Gluza \\ Institute of Physics, University of Silesia, Uniwersytecka 4, PL-40007 Katowice, Poland \\ E-mail: janusz.gluza@us.edu.pl
}

\section{Marek Gluza}

AGH - University of Science and Technology, Faculty of Physics and Applied Computer Science, al. A. Mickiewicza, 30-059 Kraków, Poland

\section{Tord Riemann}

Deutsches Elektronen-Synchrotron DESY, Platanenallee 6, 15738 Zeuthen, Germany

E-mail: Tord.Riemanndesy.de

\section{Radomir Sevillano}

Institute of Physics, University of Silesia, Uniwersytecka 4, PL-40007 Katowice, Poland

E-mail: radomir.sevillano-borkowskidus.edu.pl

\begin{abstract}
A new approach for the reduction of tensor integrals is described. The standard decomposition à la Davydychev is applied. Integrals with higher indices are then expressed in terms of scalar higher-dimensional integrals with generic indices. The approach allows to perform contractions with external momenta in a particularly efficient manner. This is due to the possibility to perform analytically the resulting sums over the indices of products of signed minors and scalar products of chords. Advantages of this approach for the calculation of cross sections are described. We are preparing the numerical package OLEC (One Loop External Contractions) with interfaces in Mathematica for algebraic and in $\mathrm{C}++$ for numerical calculations. First numerical results are discussed.
\end{abstract}

Loops and Legs in Quantum Field Theory - 11th DESY Workshop on Elementary Particle Physics April 15-20, 2012

Wernigerode, Germany

\footnotetext{
* Speaker.
} 


\section{Introduction}

Starting from the one-loop level, calculations of the squared amplitudes in Quantum Field Theory bring us to consider $n$-point tensor integrals of rank $R,(n, R)$-integrals

$$
I_{n}^{\mu_{1} \cdots \mu_{R}}=\int \frac{d^{d} k}{i \pi^{d / 2}} \frac{\prod_{r=1}^{R} k^{\mu_{r}}}{\prod_{j=1}^{n} c_{j}^{v_{j}}}
$$

where $d=4-2 \varepsilon$ and denominators $c_{j}$ have indices $v_{j}$ and chords $q_{j}$

$$
c_{j}=\left(k-q_{j}\right)^{2}-m_{j}^{2}+i \varepsilon .
$$

There are many strategies how to solve such tensor integrals, see e.g. [1 -4] and references therein..

Our procedure and notations are described in $[5,6]$ and in references quoted therein. There are two main steps which are applied to (1.1). First, higher dimensional scalar integrals are used. This is Davydychev's decomposition [7], e.g. for a $R=2$ tensor with a general number of external legs $n$

$$
I_{n}^{\mu v}=\int^{d} k^{\mu} k^{v} \prod_{r=1}^{n} c_{r}^{-1}=\sum_{i, j=1}^{n} q_{i}^{\mu} q_{j}^{v} v_{i j} I_{n, i j}^{[d+]^{2}}-\frac{1}{2} g^{\mu v} I_{n}^{[d+]} .
$$

We use the notation

$$
[d+]^{l}=d+2 l .
$$

Second, recursive relations as derived in $[8,9]$ bring the expression into a simpler form, e.g. explicitly for pentagons

$$
V_{i j} I_{5, i j}^{[d+]^{2}}=-\frac{\left(\begin{array}{l}
0 \\
j
\end{array}\right)_{5}}{()_{5}} I_{5, i}^{[d+]}+\sum_{s=1, s \neq i}^{5} \frac{\left(\begin{array}{l}
s \\
j
\end{array}\right)_{5}}{()_{5}} I_{4, i}^{[d+], s}+\frac{\left(\begin{array}{l}
i \\
j
\end{array}\right)_{5}}{()_{5}} I_{5}^{[d+]} .
$$

As may be seen, there is a decrease in both dimension and indices. Finally, recursively, we go down to basic scalar integrals in higher dimension - but at the price of appearance of inverse Gram determinants ()$_{5}$ in (1.5). It is known that inverse Gram determinants can cause trouble in realistic physical applications, because there are kinematical regions where they can be small or even vanish.

Here, the main aspect of the new approach comes into action: After insertion of (1.5) into (1.3), the chords $q_{i}, q_{j}$ are contracted with external momenta (expressed as simple differences of chords $\left.q_{a}, q_{b}\right)$. One observes the appearance of "auxiliary vectors"

$$
Q_{s}^{v}=\sum_{j=1}^{5} q_{j}^{v} \frac{\left(\begin{array}{l}
s \\
i
\end{array}\right)_{5}}{()_{5}}, \quad s=0, \ldots, 5 .
$$

Contractions of the vectors with a chord $q_{a}$ immediately eliminate the unwanted inverse of ()$_{5}$. At first glance this is really surprising and we want to stress this property in particular. If we would arrange cancellations of inverse Gram determinants ()$_{5}$ in (1.3) in a standard way, much more effort is needed $[10,11,5]$. 
To be more precise, let us give a manifest example, taken from subsection 2.1:

$$
\left(q_{a} \cdot Q_{s}\right)=\sum_{j=1}^{n-1}\left(q_{a} \cdot q_{j}\right) \frac{\left(\begin{array}{l}
s \\
j
\end{array}\right)_{n}}{()_{n}}=\frac{1}{2}\left(\delta_{a s}-\delta_{n s}\right), \quad s=1, \ldots, 5 . .
$$

The $Q$ vectors, when contracted with a chord in (1.7), give just Kronecker $\delta$-functions. The inverse Gram determinant ()$_{5}$, present in intermediate steps, disappears automatically.

Let us account now the main advantages of the new approach where chords are contracted with $Q$ vectors (signed minors):

1. Cancellation of inverse Gram determinants ()$_{5}$.

Explained as above - occasionally also cancellation by subtraction.

2. Elimination of the metric tensor $g_{\mu \nu}$.

The integral (1.3) in its original form includes the $g_{\mu \nu}$ metric tensor.

The identity (valid in $d=4$ dimensions)

$$
\frac{1}{2} g^{\mu v}=\sum_{i, j=1}^{5} \frac{\left(\begin{array}{l}
i \\
j
\end{array}\right)_{5}}{()_{5}} q_{i}^{\mu} q_{j}^{v}
$$

introduces the inverse of ()$_{5}$ again. So, as argued before, it should cancel out. In fact, e.g. for pentagons $(n=5)$,

$$
I_{5}^{\mu v}=\sum_{i, j=1}^{4} q_{i}^{\mu} q_{j}^{v} v_{i j} I_{5, i j}^{[d+]^{2}}-\frac{1}{2} g^{\mu v} I_{5}^{[d+]},
$$

and the last term in (1.5) cancels against the $g^{\mu v}$ term in (1.9). After the $g^{\mu v}$ cancellation and the contractions with chords, the remaining inverse of ()$_{5}$, which was produced in intermediate steps, disappears as well.

3. Presence of Kronecker $\delta$-functions and simple kinematical factors $Y$.

This is probably the main feature of the approach. Due to relations like (1.7) and

$$
\left(q_{a} \cdot Q_{0}\right) \equiv \sum_{j=1}^{n-1}\left(q_{a} \cdot q_{j}\right) \frac{\left(\begin{array}{l}
0 \\
j
\end{array}\right)_{n}}{()_{n}}=-\frac{1}{2}\left(Y_{a n}-Y_{n n}\right),
$$

where $Y_{i j}=-\left(q_{i}-q_{j}\right)^{2}+m_{i}^{2}+m_{j}^{2}$ are simple kinematical functions, the expressions for contracted tensor integrals presented in the next section take a simple form. Take as an example the (2.33) from the next section. For given indices $a, b$ and $t$, most of the terms vanish.

The features are independent of multiplicity (i.e. the number of external legs) and rank of the tensor integrals under consideration.

\section{The contractions}

We consider now 5-point functions and eliminate the inverse 5-point Gram determinants ()$_{5}$. Inverse 4-point Gram determinants $\left(\begin{array}{l}s \\ s\end{array}\right)_{5}$ will be treated separately. The explicit formulae cover tensor integrals until rank $R=3$. 


\subsection{The vector integral: $I_{5}^{\mu}$}

The vector integral may be written in terms of the scalar 5-point function $E$ and 4-point scalar functions $I_{4}^{s}$, where $s$ indicates the scratched line of the 5-point topology:

$$
I_{5}^{\mu}=E Q_{0}^{\mu}-\sum_{s=1}^{5} I_{4}^{s} Q_{s}^{\mu}, \quad E=\frac{1}{\left(\begin{array}{l}
0 \\
0
\end{array}\right)_{5}} \sum_{s=1}^{5}\left(\begin{array}{l}
s \\
0
\end{array}\right)_{5} I_{4}^{s} .
$$

For $q_{n}=0, \quad a=1, \ldots, n-1, \quad s=1, \ldots n$, the multiplication with chords introduces (1.7) and (1.10), and the final expression for a contraction of the vector integral $I_{5}^{\mu}$ with an arbitrary chord $q_{a, \mu}$ becomes

$$
\begin{aligned}
\left(q_{a} \cdot I_{5}\right) & =E\left(q_{a} \cdot Q_{0}\right)-\sum_{s=1}^{5} I_{4}^{s}\left(q_{a} \cdot Q_{s}\right) \\
& =-\frac{1}{2} E\left(Y_{a 5}-Y_{55}\right)-\frac{1}{2} \sum_{s=1}^{5} I_{4}^{s}\left(\delta_{a s}-\delta_{5 s}\right) .
\end{aligned}
$$

Here we have applied (1.7) and (1.10) for $n=5$. In fact no inverse Gram determinants have appeared.

2.2 The tensor integrals of $\operatorname{rank} R=2: \quad I_{5}^{\mu v}$

From equation (3.5) of [5] one gets

$$
I_{5}^{\mu v}=I_{5}^{\mu} Q_{0}^{v}-\sum_{s=1}^{5} I_{4}^{\mu, s} Q_{s}^{v},
$$

with

$$
I_{4}^{\mu, s}=-\sum_{i=1}^{4} q_{i}^{\mu} I_{4, i}^{[d+], s}=Q_{0}^{s, \mu} I_{4}^{s}-\sum_{t=1}^{5} Q_{t}^{s, \mu} I_{3}^{s t} .
$$

There are two kinds of contractions to be considered: with the metric tensor or with two chords. In principle, one may also meet in chiral theories contractions of the form

$$
q^{a \alpha} q^{b \beta} \varepsilon_{\alpha \beta \mu \nu} I_{5}^{\mu v}
$$

Proper organization of the matrix element evaluation will exclude the appearance of such terms since cross sections are either helicity and spin summed and thus pure scalar quantities or written in terms of helicity states. In the latter case, the scalar factors with the loop functions are also free of $\gamma_{5}$, and the $\varepsilon$ tensor might influence the matrix element basis. Nevertheless, we add one rank $R=2$ example in order to demonstrate that this kind of sums may also be treated.

2.2.1 Contractions with chords: $q_{a \mu} q_{b v} I_{5}^{\mu v}$

By contraction, (2.3) rewrites into

$$
q_{a \mu} q_{b v} I_{5}^{\mu v}=\left(q_{a} \cdot I_{5}\right)\left(q_{b} \cdot Q_{0}\right)-\sum_{s=1}^{5}\left(q_{a} \cdot I_{4}^{s}\right)\left(q_{b} \cdot Q_{s}\right) .
$$


Here no inverse ()$_{5}$ occurs anymore. Inserting the sums $\Sigma_{a}^{2, s}$ and $\Sigma_{a}^{1, s t}$ from $[6],\left(q_{a} \cdot I_{4}^{s}\right)$ can be written as

$$
\left(q_{a} \cdot I_{4}^{s}\right)=\frac{1}{\left(\begin{array}{l}
s \\
s
\end{array}\right)_{5}}\left[\Sigma_{a}^{2, s} I_{4}^{s}-\Sigma_{a}^{1, s t} I_{3}^{s t}\right]=-\frac{1}{2}\left\{\left(Y_{a 5}-Y_{55}\right) I_{4}^{s}+\sum_{t=1}^{5}\left(\delta_{a t}-\delta_{5 t}\right) I_{3}^{s t}+\left(\delta_{a s}-\delta_{5 s}\right) R^{s}\right\}
$$

where we introduced the abbreviation

$$
R^{s} \equiv \frac{1}{\left(\begin{array}{l}
s \\
s
\end{array}\right)_{5}}\left[\left(\begin{array}{l}
s \\
0
\end{array}\right)_{5} I_{4}^{s}-\sum_{t=1}^{5}\left(\begin{array}{l}
s \\
t
\end{array}\right)_{5} I_{3}^{s t}\right]=\frac{1}{\left(\begin{array}{l}
0 s \\
0 s
\end{array}\right)_{5}}\left[\left(\begin{array}{l}
s \\
0
\end{array}\right)_{5} I_{4}^{[d+], s}-\sum_{t=1}^{5}\left(\begin{array}{c}
0 s \\
0 t
\end{array}\right)_{5} I_{3}^{s t}\right]
$$

The $2^{\text {nd }}$ representation of $R^{s}$ in (2.8) can be used if the four-point Gram determinant $\left(\begin{array}{l}s \\ s\end{array}\right)_{5}$ is small or even vanishes.

\subsubsection{Contractions with the metric tensor: $g_{\mu v} I_{5}^{\mu v}$}

The second scalar which can be constructed from the tensor of rank 2 is $g_{\mu v} I_{5}^{\mu v}$. Due to (2.3) we have to evaluate the following scalar products:

$$
\begin{aligned}
& \left(Q_{0} \cdot Q_{0}\right)=\frac{1}{2}\left[\frac{\left(\begin{array}{l}
0 \\
0
\end{array}\right)_{5}}{()_{5}}+Y_{55}\right], \\
& \left(Q_{0} \cdot Q_{s}\right)=\frac{1}{2}\left[\frac{\left(\begin{array}{l}
s \\
0
\end{array}\right)_{5}}{()_{5}}-\delta_{s 5}\right], \\
& \left(Q_{0}^{s} \cdot Q_{s}\right)=-\frac{1}{2} \delta_{s 5}, \\
& \left(Q_{t}^{s} \cdot Q_{s}\right)=0 .
\end{aligned}
$$

In this case the terms with $\frac{1}{()_{5}}$ cancel and, not surprisingly, the result finally is

$$
g_{\mu v} I_{5}^{\mu v}=\frac{Y_{55}}{2} E+I_{4}^{5}
$$

2.2.3 Contractions with the antisymmetric tensor: $q^{a \mu} q^{b v} \varepsilon_{\mu v \alpha \beta} I_{5}^{\alpha \beta}$

A reduction of pseudoscalar contractions leads to expressions of the type

$$
P[C]=q_{a}^{\rho} q_{b}^{\lambda} \varepsilon_{\mu v \rho \lambda} \sum_{i, j=1}^{4} q_{i}^{\mu} q_{j}^{v} C_{i j} .
$$

This may be evaluated as follows:

$$
\begin{aligned}
P[C] & =q_{a}^{\rho} q_{b}^{\lambda} \varepsilon_{\mu v \rho \lambda} g_{\mu^{\prime}}^{\mu} g_{v^{\prime}} \sum_{i, j=1}^{4} q_{i}^{\mu^{\prime}} q_{j}^{v^{\prime}} C_{i j} \\
& =\frac{4}{()_{5}^{2}} q_{a}^{\rho} q_{b}^{\lambda} \varepsilon_{\mu v \rho \lambda} \sum_{k, l=1}^{4}\left(\begin{array}{c}
k \\
l
\end{array}\right)_{5} q_{k}^{\mu} q_{l, \mu^{\prime}} \sum_{m, n=1}^{4}\left(\begin{array}{c}
m \\
n
\end{array}\right)_{5} q_{m}^{v} q_{n, v^{\prime}} \sum_{i, j=1}^{4} q_{i}^{\mu^{\prime}} q_{j}^{v^{\prime}} C_{i j} \\
& =\frac{4}{()_{5}^{2}} \sum_{a+b+m=1}^{4}\left[q_{a}^{\rho} q_{b}^{\lambda} \varepsilon_{\mu v \rho \lambda} q_{k}^{\mu} q_{m}^{v}\right] \sum_{l, n=1}^{4}\left(\begin{array}{c}
k \\
l
\end{array}\right)\left(\begin{array}{c}
m \\
n
\end{array}\right) \sum_{5 i, j=1}^{4}\left(q_{l} \cdot q_{i}\right)\left(q_{n} \cdot q_{j}\right) C_{i j} \\
& =\frac{4}{()_{5}^{2}} \operatorname{det}\left[q_{1}, q_{2}, q_{3}, q_{4}\right] \sum_{\substack{k, m=1 \\
a+b+k+m=10}}^{4} S(a, b, k, m) \sum_{l, n=1}^{4}\left(\begin{array}{c}
k \\
l
\end{array}\right)_{5}\left(\begin{array}{c}
m \\
n
\end{array}\right) \sum_{5}[C]
\end{aligned}
$$


where $S(a, b, k, m)$ gives the sign of permutations needed to place the indices in increasing order. Further we introduced the abbreviation

$$
\Sigma_{l n}[C]=\sum_{i, j=1}^{4}\left(q_{l} \cdot q_{i}\right)\left(q_{n} \cdot q_{j}\right) C_{i j}
$$

the calculation of which has to be performed for specific cases.

Let us choose for demonstrational purposes $C_{i j}=\left(\begin{array}{l}i s \\ j s\end{array}\right)_{5}$ :

$$
\begin{aligned}
\Sigma_{l n}[C] & =\frac{1}{2}\left(q_{l} \cdot q_{n}\right)\left(\begin{array}{l}
s \\
s
\end{array}\right)_{5}-\frac{1}{4}()_{5}\left(\delta_{l s}-\delta_{5 s}\right)\left(\delta_{n s}-\delta_{5 s}\right) \\
& = \begin{cases}\frac{1}{2}\left(q_{l} \cdot q_{n}\right)\left(\begin{array}{l}
s \\
s
\end{array}\right)_{5}-\frac{1}{4}()_{5} \delta_{l s} \delta_{n s} & \mathrm{~s} \neq 5, \\
\frac{1}{2}\left(q_{l} \cdot q_{n}\right)\left(\begin{array}{l}
s \\
s
\end{array}\right)_{5}-\frac{1}{4}()_{5} & \mathrm{~s}=5 .\end{cases}
\end{aligned}
$$

Next we have to evaluate the sum $\sum_{l, n=1}^{4}\left(\begin{array}{l}k \\ l\end{array}\right)_{5}\left(\begin{array}{c}m \\ n\end{array}\right)_{5} \cdot \Sigma_{l n}[C]$. Taking the first term in (2.14), to begin with, we have

$$
\begin{aligned}
\frac{1}{2} \sum_{l, n=1}^{4}\left(q_{l} \cdot q_{n}\right)\left(\begin{array}{l}
k \\
l
\end{array}\right)_{5}\left(\begin{array}{l}
m \\
n
\end{array}\right)_{5} & =\frac{1}{2} \sum_{l=1}^{4}\left(\begin{array}{l}
k \\
l
\end{array}\right)_{5} \sum_{n=1}^{4}\left(q_{l} \cdot q_{n}\right)\left(\begin{array}{l}
m \\
n
\end{array}\right)_{5} \\
& =\frac{1}{4} \sum_{l=1}^{4}\left(\begin{array}{l}
k \\
l
\end{array}\right)_{5} \delta_{m l}()_{5} \\
& =\frac{()_{5}}{4}\left(\begin{array}{l}
k \\
m
\end{array}\right)_{5}
\end{aligned}
$$

and for $s \neq 5$ we thus have

$$
\begin{aligned}
\sum_{l, n=1}^{4}\left(\begin{array}{l}
k \\
l
\end{array}\right)_{5}\left(\begin{array}{l}
m \\
n
\end{array}\right)_{5} \cdot \Sigma_{l n}[C] & =\frac{()_{5}}{4}\left[\left(\begin{array}{c}
k \\
m
\end{array}\right)_{5}\left(\begin{array}{l}
s \\
s
\end{array}\right)_{5}-\left(\begin{array}{l}
k \\
s
\end{array}\right)_{5}\left(\begin{array}{c}
m \\
s
\end{array}\right)_{5}\right] \\
& =\frac{()_{5}^{2}}{4}\left(\begin{array}{c}
k s \\
m s
\end{array}\right)_{5} .
\end{aligned}
$$

Similarly we have for $s=5$

$$
\sum_{l, n=1}^{4}\left(\begin{array}{l}
k \\
l
\end{array}\right)_{5}\left(\begin{array}{l}
m \\
n
\end{array}\right)_{5} \cdot \Sigma_{l n}[C]=\frac{()_{5}}{4}\left[\left(\begin{array}{l}
k \\
m
\end{array}\right)_{5}\left(\begin{array}{l}
5 \\
5
\end{array}\right)_{5}-\sum_{l, n=1}^{4}\left(\begin{array}{l}
k \\
l
\end{array}\right)_{5}\left(\begin{array}{l}
m \\
n
\end{array}\right)_{5}\right] .
$$

Due to

$$
\sum_{l=1}^{5}\left(\begin{array}{l}
k \\
l
\end{array}\right)_{5}=0, \text { i.e. } \sum_{l=1}^{4}\left(\begin{array}{l}
k \\
l
\end{array}\right)_{5}=-\left(\begin{array}{l}
k \\
5
\end{array}\right)_{5}
$$

we get for $s=5$ the same result as in (2.16).

Thus the total result reads for the specific choice of $C_{i j}$ ):

$$
P\left[\left(\begin{array}{c}
i s \\
j s
\end{array}\right)_{5}\right]=\operatorname{det}\left[q_{1}, q_{2}, q_{3}, q_{4}\right] \sum_{\substack{k, m=1 \\
a+b+k+m=10}}^{4} S(a, b, k, m)\left(\begin{array}{c}
k s \\
m s
\end{array}\right)_{5} .
$$


In the summation of (2.19) all indices $a, b, k, m$ must be different, i.e. the sum contains only 2 terms, with $k$ and $m$ exchanged. Since these terms come with opposite sign and (2.16) is symmetric in $k$ and $m$, the final result for the present example vanishes, i.e.

$$
\begin{aligned}
P\left[\left(\begin{array}{c}
i s \\
j s
\end{array}\right)_{5}\right] & =q_{a}^{\rho} q_{b}^{\lambda} \varepsilon_{\mu v \rho \lambda} \sum_{i, j=1}^{4} q_{i}^{\mu} q_{j}^{v}\left(\begin{array}{c}
i s \\
j s
\end{array}\right)_{5} \\
& =0 .
\end{aligned}
$$

2.3 The tensors of rank $R=3: I_{5}^{\mu \nu \lambda}$

From eq.(3.5) of [5] one gets

$$
I_{5}^{\mu \nu \lambda}=I_{5}^{\mu \nu} \cdot Q_{0}^{\lambda}-\sum_{s=1}^{5} I_{4}^{\mu \nu, s} \cdot Q_{s}^{\lambda},
$$

where according to (3.12) in [5] it is

$$
\begin{gathered}
I_{4}^{\mu v, s}=I_{4}^{\mu, s} Q_{0}^{s, v}-\sum_{t=1}^{5} I_{3}^{\mu, s t} Q_{t}^{s, v}-\frac{()_{5}}{\left(\begin{array}{c}
s \\
s
\end{array}\right)_{5}} Q_{s}^{\mu} Q_{s}^{v} I_{4}^{[d+], s}, \\
I_{3}^{\mu, s t}=-\sum_{i=1}^{4} q_{i}^{\mu} I_{3, i}^{[d+], s t}=Q_{0}^{s t, \mu} I_{3}^{s t}-\sum_{u=1}^{5} Q_{u}^{s t, \mu} I_{2}^{s t u} .
\end{gathered}
$$

\subsubsection{Contractions with chords: $q_{a \mu} q_{b v} q_{c \lambda} I_{5}^{\mu \nu \lambda}$}

The contraction of (2.21) with $q_{c \lambda}$ is trivial due to (1.7) and (1.10). Further, the term $q_{a \mu} q_{b v} I_{5}^{\mu \nu}$ is known from subsection 2.3.1. The only new object to be investigated is therefore

$$
q_{a \mu} q_{b v} I_{4}^{\mu \nu, s}=\left(q_{a} \cdot I_{4}^{s}\right)\left(q_{b} \cdot Q_{0}^{s}\right)-\sum_{t=1}^{5}\left(q_{a} \cdot I_{3}^{s t}\right)\left(q_{b} \cdot Q_{t}^{s}\right)-\frac{()_{5}}{\left(\begin{array}{l}
s \\
s
\end{array}\right)_{5}}\left(q_{a} \cdot Q_{s}\right)\left(q_{b} \cdot Q_{s}\right) I_{4}^{[d+], s} .
$$

New sums may be expressed by those derived in [6]:

$$
\begin{aligned}
& \left(q_{b} \cdot Q_{0}^{s}\right)=\frac{1}{\left(\begin{array}{c}
s \\
s
\end{array}\right)_{5}} \Sigma_{b}^{2, s}, \\
& \left(q_{b} \cdot Q_{t}^{s}\right)=\frac{1}{\left(\begin{array}{l}
s \\
s
\end{array}\right)_{5}} \Sigma_{b}^{1, s t},
\end{aligned}
$$

and

$$
\begin{aligned}
\left(q_{a} \cdot I_{3}^{s t}\right) & =\frac{1}{\left(\begin{array}{c}
s t \\
s t
\end{array}\right)_{5}}\left[\Sigma_{a}^{3, s t} I_{3}^{s t}-\sum_{u=1}^{5} \Sigma_{a}^{1, s t u} I_{2}^{s t u}\right] \\
& =-\frac{1}{2}\left\{\left(Y_{a 5}-Y_{55}\right) I_{3}^{s t}+\sum_{u=1}^{5}\left(\delta_{a u}-\delta_{5 u}\right) I_{2}^{s t u}+\left(\delta_{a s}-\delta_{5 s}\right) R^{s t}+\left(\delta_{a t}-\delta_{5 t}\right) R^{t s}\right\}
\end{aligned}
$$

where we introduced as a further abbreviation

$$
\begin{aligned}
R^{s t} & \equiv \frac{1}{\left(\begin{array}{c}
s t \\
s t
\end{array}\right)_{5}}\left[\left(\begin{array}{c}
s t \\
0 t
\end{array}\right)_{5} I_{3}^{s t}-\sum_{u=1}^{5}\left(\begin{array}{c}
s t \\
u t
\end{array}\right)_{5} I_{2}^{s t u}\right] \\
& =\frac{1}{\left(\begin{array}{c}
0 s t \\
0 s t
\end{array}\right)_{5}}\left[\left(\begin{array}{c}
s t \\
0 t
\end{array}\right)_{5}(d-2) I_{3}^{[d+], s t}-\sum_{u=1}^{5}\left(\begin{array}{c}
0 s t \\
0 u t
\end{array}\right)_{5} I_{2}^{s t u}\right] .
\end{aligned}
$$


From

$$
\begin{aligned}
& \Sigma_{b}^{2, s}=-\frac{1}{2}\left\{\left(\begin{array}{l}
s \\
s
\end{array}\right)_{5}\left(Y_{b 5}-Y_{55}\right)+\left(\begin{array}{l}
s \\
0
\end{array}\right)_{5}\left(\delta_{b s}-\delta_{5 s}\right)\right\}, \\
& \Sigma_{b}^{1, s t}=\frac{1}{2}\left\{\left(\begin{array}{l}
s \\
s
\end{array}\right)_{5}\left(\delta_{b t}-\delta_{5 t}\right)-\left(\begin{array}{l}
s \\
t
\end{array}\right)_{5}\left(\delta_{b s}-\delta_{5 s}\right)\right\},
\end{aligned}
$$

multiplying (2.7) and (2.26), respectively, we see that the $\left(\delta_{b s}-\delta_{5 s}\right)$-terms in (2.29) combine with the first terms in (2.7) and (2.26) to yield the contribution

$$
\frac{1}{4}\left(Y_{a 5}-Y_{55}\right)\left(\delta_{b s}-\delta_{5 s}\right) R^{s}
$$

Collecting all contributions, the following relation is useful in order to to eliminate some $\frac{1}{\left(\begin{array}{c}s \\ s\end{array}\right)_{5}}$-terms:

$$
R^{s t}+\frac{\left(\begin{array}{l}
s \\
t
\end{array}\right)_{5}}{\left(\begin{array}{l}
s \\
s
\end{array}\right)_{5}} R^{t s}=\frac{\left(\begin{array}{l}
s \\
0
\end{array}\right)_{5}}{\left(\begin{array}{l}
s \\
s
\end{array}\right)_{5}} I_{3}^{s t}-\sum_{u=1}^{5} \frac{\left(\begin{array}{l}
s \\
u
\end{array}\right)_{5}}{\left(\begin{array}{l}
s \\
s
\end{array}\right)_{5}} I_{2}^{s t u}
$$

The result for (2.23) is explicitly symmetric in the indices $a, b$ :

$$
q_{a \mu} q_{b v} I_{4}^{\mu \nu, s}=q_{a \mu} q_{b v} \bar{I}_{4}^{\mu \nu, s}-\frac{1}{4}\left(\delta_{a s}-\delta_{5 s}\right)\left(\delta_{b s}-\delta_{5 s}\right) \frac{()_{5}}{\left(\begin{array}{l}
s \\
s
\end{array}\right)_{5}} I_{4}^{[d+], s},
$$

and the first term on the right hand side is

$$
\begin{aligned}
q_{a \mu} q_{b v} \bar{I}_{4}^{\mu v, s}= & \frac{1}{4}\left(Y_{a 5}-Y_{55}\right)\left(Y_{b 5}-Y_{55}\right) \cdot I_{4}^{s} \\
& +\frac{1}{4}\left(\delta_{a s}-\delta_{5 s}\right)\left(\delta_{b s}-\delta_{5 s}\right) \frac{1}{\left(\begin{array}{l}
s \\
s
\end{array}\right)_{5}}\left[\left(\begin{array}{c}
s \\
0
\end{array}\right)_{5} R^{s}-\sum_{t=1}^{5}\left(\begin{array}{l}
s \\
t
\end{array}\right)_{5} R^{s t}\right] \\
& +\frac{1}{4}\left[\left(\delta_{a s}-\delta_{5 s}\right)\left(Y_{b 5}-Y_{55}\right)+\left(Y_{a 5}-Y_{55}\right)\left(\delta_{b s}-\delta_{5 s}\right)\right] R^{s} \\
& +\frac{1}{4} \sum_{t=1}^{5}\left[\left(\delta_{a s}-\delta_{5 s}\right)\left(\delta_{b t}-\delta_{5 t}\right)+\left(\delta_{b s}-\delta_{5 s}\right)\left(\delta_{a t}-\delta_{5 t}\right)\right] R^{s t} \\
& +\frac{1}{4} \sum_{t=1}^{5}\left[\left(\delta_{a t}-\delta_{5 t}\right)\left(Y_{b 5}-Y_{55}\right)+\left(Y_{a 5}-Y_{55}\right)\left(\delta_{b t}-\delta_{5 t}\right)\right] I_{3}^{s t} \\
& +\frac{1}{4} \sum_{t=1}^{5}\left(\delta_{a t}-\delta_{5 t}\right)\left(\delta_{b t}-\delta_{5 t}\right) R^{t s}+\frac{1}{4} \sum_{t, u=1}^{5}\left(\delta_{b t}-\delta_{5 t}\right)\left(\delta_{a u}-\delta_{5 u}\right) I_{2}^{s t u} .
\end{aligned}
$$

It is worth mentioning that the second term in (2.32) may be written as

$$
()_{5} I_{4}^{[d+], s}=\left(\begin{array}{l}
0 \\
0
\end{array}\right)_{5} I_{4}^{s}-\sum_{t=1}^{5}\left(\begin{array}{l}
t \\
0
\end{array}\right)_{5} I_{3}^{s t}-\left(\begin{array}{l}
s \\
0
\end{array}\right)_{5} R^{s}
$$

The $R^{s}, R^{s t}, \sum_{t=1}^{5}\left(\begin{array}{l}s \\ t\end{array}\right){ }_{5} R^{s t}$ and the scalar integrals in (2.33) are independent of the indices $a, b, c$ and can be considered as buildung blocks. The summation over $s, t, u$ is simplified by the explicit appearance of the Kronecker $\delta$-functions, i.e. all contractions are just given in terms of different combinations of the buildung blocks. 
There still occur contributions with factor $\frac{1}{\left(s_{s}^{s}\right)_{5}}$ in (2.32) and in (2.33). At first we observe that this contribution is indeed quite "localized": in general one will be able to avoid the small $\left(\begin{array}{l}s \\ s\end{array}\right)_{5}$ to appear on line $s=5$ by choosing the integration momenta properly. Let say $\left(\begin{array}{l}s_{0} \\ s_{0}\end{array}\right)_{5}$ is small. Then the critical contribution will only occur if $a=b=c=s_{0}$, i.e. only in a single contraction.

Nevertheless, if one wants to avoid the $\frac{1}{\left(\begin{array}{c}s \\ s\end{array}\right)_{5}}$ completely, one can express the corresponding contribution for the sum of these terms in (2.32) and (2.33) in terms of higher dimensional integrals, making again use of (2.8). Let us call the sum $J_{4}^{S}$ :

$$
\begin{aligned}
J_{4}^{s} & \equiv \frac{1}{\left(\begin{array}{l}
s \\
s
\end{array}\right)_{5}}\left\{-()_{5} I_{4}^{[d+], s}+\left(\begin{array}{l}
s \\
0
\end{array}\right)_{5} R^{s}-\sum_{t=1}^{5}\left(\begin{array}{l}
s \\
t
\end{array}\right)_{5} R^{s t}\right\} \\
& =\frac{-1}{\left(\begin{array}{l}
0 s \\
0 s
\end{array}\right)_{5}}\left\{()_{5}(d-2)(d-1) I_{4}^{[d+]^{2}, s}-\left(\begin{array}{l}
0 \\
0
\end{array}\right)_{5} I_{4}^{[d+], s}+\sum_{t=1}^{5}\left(\begin{array}{l}
t \\
0
\end{array}\right)_{5}(d-2) I_{3}^{[d+], s t}+\sum_{t=1}^{5}\left(\begin{array}{c}
0 s \\
0 t
\end{array}\right)_{5} R^{s t}\right\} .
\end{aligned}
$$

The higher dimensional integrals can then be evaluated as described in [5].

Let us call the second term on the right hand side of (2.21) $C_{5, a b c}$. It becomes, when contracted with momenta $q_{a}, q_{b}$ and $q_{c}$ :

$$
\begin{aligned}
C_{5, a b c} & =-q_{a \mu} q_{b v} q_{c \lambda} \sum_{s=1}^{5} I_{4}^{\mu v, s} \cdot Q_{s}^{\lambda} \\
& =-\sum_{s=1}^{5} q_{a \mu} q_{b v} I_{4}^{\mu v, s} \cdot \frac{1}{2}\left(\delta_{c s}-\delta_{5 s}\right) .
\end{aligned}
$$

The last term again contains (2.32). Introducing $\mathrm{Y}_{a}=Y_{a 5}-Y_{55}$ and making use of (2.35), we may collect terms and get

$$
\begin{aligned}
C_{5, a b c}=\frac{1}{8} \quad\{ & J_{4}^{5}-\delta_{a b} \delta_{a c} J_{4}^{a}+\mathrm{Y}_{a} \mathrm{Y}_{b}\left(I_{4}^{5}-I_{4}^{c}\right)+\mathrm{Y}_{a}\left(I_{3}^{b 5}+I_{3}^{c 5}-I_{3}^{b c}\right)+\mathrm{Y}_{b}\left(I_{3}^{a 5}+I_{3}^{c 5}-I_{3}^{a c}\right) \\
& +I_{2}^{a b 5}+I_{2}^{a c 5}+I_{2}^{b c 5}-I_{2}^{a b c}-\mathrm{Y}_{a}\left(R^{5}+\delta_{b c} R^{b}\right)-\mathrm{Y}_{b}\left(R^{5}+\delta_{a c} R^{a}\right) \\
& \left.-R^{5 a}-R^{5 b}-R^{5 c}+\delta_{a b}\left(R^{a 5}-R^{a c}\right)+\delta_{a c}\left(R^{a 5}-R^{a b}\right)+\delta_{b c}\left(R^{b 5}-R^{b a}\right)\right\}
\end{aligned}
$$

2.3.2 Contractions with the metric tensor: $g_{\mu \nu} q_{a \lambda} I_{5}^{\mu \nu \lambda}$

In order to calculate $g_{\mu v} I_{5}^{\mu \nu \lambda}$, we need $g_{\mu v} I_{4}^{\mu v}$ and thus the further scalar products, see (2.22):

$$
\begin{aligned}
& \left(Q_{0}^{s} \cdot Q_{0}^{s}\right)=\frac{1}{2\left(\begin{array}{l}
s \\
s
\end{array}\right)_{5}}\left[\left(\begin{array}{c}
0 s \\
0 s
\end{array}\right)_{5}+2\left(\begin{array}{l}
s \\
0
\end{array}\right)_{5} \delta_{s 5}\right]+\frac{1}{2} Y_{55}, \\
& \left(Q_{s} \cdot Q_{s}\right)=\frac{1}{2} \frac{\left(\begin{array}{l}
s \\
s
\end{array}\right)_{5}}{()_{5}} \\
& \left(Q_{t}^{s} \cdot Q_{0}^{s}\right)=\frac{1}{2\left(\begin{array}{l}
s \\
s
\end{array}\right)_{5}}\left[\left(\begin{array}{c}
t s \\
0 s
\end{array}\right)_{5}-\left(\begin{array}{l}
s \\
s
\end{array}\right)_{5} \delta_{t 5}+\left(\begin{array}{l}
s \\
t
\end{array}\right)_{5} \delta_{s 5}\right], \\
& \left(Q_{t}^{s} \cdot Q_{0}^{s t}\right)=\frac{1}{2\left(\begin{array}{l}
s \\
s
\end{array}\right)_{5}}\left[\quad-\left(\begin{array}{l}
s \\
s
\end{array}\right)_{5} \delta_{t 5}+\left(\begin{array}{l}
s \\
t
\end{array}\right)_{5} \delta_{s 5}\right], \\
& \left(Q_{t}^{s} \cdot Q_{u}^{s t}\right)=0
\end{aligned}
$$


which yields

$$
g_{\mu v} I_{4}^{\mu v, s}=\frac{Y_{55}}{2} I_{4}^{s}+I_{3}^{s 5}+\delta_{s 5} R^{s}
$$

and finally

$$
g_{\mu \nu} q_{a \lambda} I_{5}^{\mu \nu \lambda}=-\frac{Y_{55}}{4}\left[\left(Y_{a 5}-Y_{55}\right) E+I_{4}^{a}-I_{4}^{5}\right]-\frac{1}{2}\left[\left(Y_{a 5}-Y_{55}\right) I_{4}^{5}+I_{3}^{a 5}-R^{5}\right] .
$$

It is remarkable that (2.39) is trivial again for $s \neq 5$. For $s=5$, however, the standard cancellation of propagators does not work and for this case (2.39) is indeed a useful result.

\section{The OLEC package - first numerical results}

The relations introduced in the previous sections have been implemented in Mathematica. A corresponding file OLECv0.9.m can be found at [12]. Certainly, computer algebra programs like Mathematica are not the optimal and efficient tools for ultimate numerical applications, they are slow. However, there are some reasons to start with Mathematica. First, standard tensor reductions for five and six point functions discussed in [11] have been also implemented in the Mathematica package hexagon.m [13, 14]. The new method of direct contractions of tensors as introduced in $[6,5]$ and presented here can be compared with hexagon.m. Second, if basic scalar integrals are known analytically, then the complete reductions are automatically given also in an analytic form. This approach could be used for instance to investigate infrared structure of amplitudes $[15-17,11]$. Of course, for numerical applications one will prefer packages written in Fortran or C++. For efficient reductions of five-point tensor integrals, see V. Yundin's C++ package PJFry [18-20]).

For the contracted 5-point functions with which we are dealing here, the following notation is used: CEx (chords), where "C" stands for "contracted" and "E" stands traditionally for pentagons. The argument "chords" represents a list of indices, and their length depends on the rank "x" of the considered object. In this way, $C E 1(a)$ is equivalent to $(2.2), C E 2(a, b)$ is equivalent to (2.6) and $C E 3(a, b, c)$ is equivalent to appropriate relations given in subsection 2.3.1. For the objects contracted with the metric tensor $g_{\mu v}$ we have two objects up to rank 3: $C E 2 g$ and $C E 3 g(a)$. They correspond to (2.10) and (2.40), respectively.

Now, we can compare these objects with corresponding objects which come from standard reductions. Below we will use the notation of LoopTools/FF [21, 22] for coefficients of corresponding tensorial objects $E 0 i$ :

$$
\begin{aligned}
C E 1(a) \equiv & q_{a}^{\mu_{1}}\left\{\sum_{i=1}^{4} q_{\mu_{1}}^{i} E 0 i[e e i, i n v s]\right\}, \\
C E 2(a, b) \equiv & q_{a}^{\mu_{1}} q_{b}^{\mu_{2}}\left\{\sum_{\{i, j\}=1}^{4} q_{\mu_{1}}^{i} q_{\mu_{2}}^{j} E 0 i[e e i j, i n v s]+g_{\mu_{1} \mu_{2}} E 0 i[e e 00, i n v s]\right\}, \\
C E 3(a, b, c) \equiv & q_{a}^{\mu_{1}} q_{b}^{\mu_{2}} q_{c}^{\mu_{3}}\left\{\sum_{\{i, j, k\}=1}^{4} q_{\mu_{1}}^{i} q_{\mu_{2}}^{j} q_{\mu_{3}}^{j} E 0 i[e e i j k, i n v s]\right. \\
& \left.+\sum_{i=1}^{4}\left(g_{\mu_{1} \mu_{2}} q_{\mu_{3}}^{i}+g_{\mu_{1} \mu_{3}} q_{\mu_{2}}^{i}+g_{\mu_{2} \mu_{3}} q_{\mu_{1}}^{i}\right) E 0 i[e e 00 i, i n v s]\right\} .
\end{aligned}
$$


For contractions with the metric tensor:

$$
\begin{aligned}
C E 2 g & \equiv E 0 i[e e 001, i n v s], \\
C E 3 g(a) & \equiv g^{\mu_{1} \mu_{2}} q_{a}^{\mu_{3}}\left\{\sum_{\{i, j, k\}=1}^{4} q_{\mu_{1}}^{i} q_{\mu_{2}}^{j} q_{\mu_{3}}^{j} E 0 i[e e i j k, \text { invs }]\right. \\
& \left.+\sum_{i=1}^{4}\left(g_{\mu_{1} \mu_{2}} q_{\mu_{3}}^{i}+g_{\mu_{1} \mu_{3}} q_{\mu_{2}}^{i}+g_{\mu_{2} \mu_{3}} q_{\mu_{1}}^{i}\right) E 0 i[e e 00 i, i n v s]\right\} .
\end{aligned}
$$

Numerical results are shown in the file Contracts_examples.nb. They are compared for various chord indices using the Looptools/FF and OneLoop [23] packages.

Below we tabularize an example of results extracted from Contracts_examples.nb for kinematical points defined by:

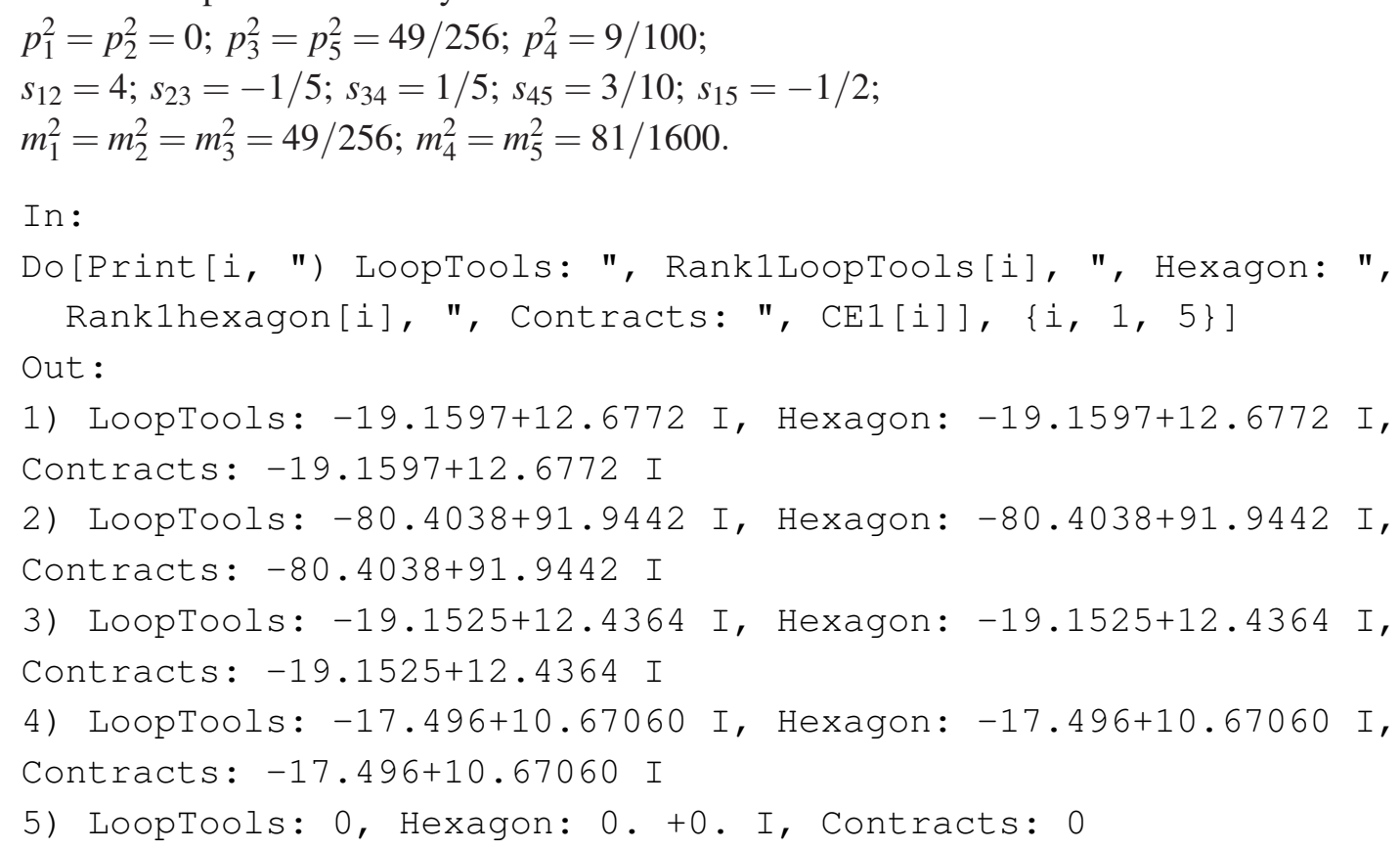

In addition, comparisons concerning the speed of calculation have been made. As expected, the Mathematica package OLEC.m v.0.9 is at least one order of magnitude faster than hexagon.m but still slower than the Fortran package LoopTools/FF.

Thus, a second package OLEC v. 0.9 written in $\mathrm{C}++$ has been prepared for numerical studies. It should be assumed as the skeleton for the first version of the ultimate package. It has to be further optimized and additional refinements for specific kinematical points are needed, similar to those which were implemented for the standard reductions within the PJFRY package.

The OLEC package can be downloaded from [12]. It is linked with both the LoopTools/FF and the OneLoop libraries. Our first package, where the contractions replace the explicit tensor reductions is at the moment comparable in speed to numerical results of 5-point tensor reductions implemented in LoopTools/FF. This has been tested for specific rank 2 tensors in (3.2) and tensors of rank 3 in (3.1). Details can be found in the module example_2 of the Makefile in [12] where 
$C E 2(1,2)$ and $C E 3(1,2,3)$ can be calculated and compared with corresponding LoopTools objects using different flags, for users convenience. The result is not too surprizing. Evidently, for higher tensor ranks the advantage of contractions compared to rank reductions, introducing multiple sums, should become more pronounced.

\section{Conclusions and outlook}

A new method of evaluation of contracted tensor integrals has been presented for five-point one loop integrals. So far individual objects like $C E 1, C E 2$ and $C E 3$ have been resolved and implemented into first codes. What remains is to apply it to the calculation of whole amplitudes for some physical processes.

In the near future the first version 1.0 of the complete code in $\mathrm{C}++$ and Fortran 90 is planned to be released. Here we have shown the backbone of the code with first numerical estimations. In addition, higher than rank 3 tensor structures for 5-point functions and tensorial integrals with more than five external legs are in preparation.

\section{Acknowledgments}

We would like to thank Andreas van Hameren, Thomas Hahn and Krzysztof Kajda for useful discussions and help. Work supported by European Initial Training Network LHCPHENOnet PITN-GA-2010-264564 and by Polish Ministry of Science and Higher Education from budget for science for 2010-2013 under grant number N N202 102638.

\section{References}

[1] Z. Bern et al., The NLO multileg working group: Summary report, 2008. Presented at 5th Les Houches Workshop on Physics at TeV Colliders, Les Houches, France, 11 - 29 June 2007. [arXiv:0803.0494].

[2] J. Andersen et al., The SM and NLO Multileg Working Group: Summary report, 2010. Proceedings of the 6th Workshop on Physics at TeV colliders, 8 - 26 June 2009, Les Houches, France. Dedicated to Thomas Binoth. [arXiv:1003.1241].

[3] J. Alcaraz Maestre et al., The SM and NLO Multileg and SM MC Working Groups: Summary Report, 2012. Report of the SM and NLO Multileg and SM MC Working Groups for the Workshop 'Physics at TeV Colliders', Les Houches, France, 31 May - 8 June, 2011. See [arXiv:1203.1488] and [arXiv:1203.6803].

[4] J. Blümlein, S. Moch, and Tord Riemann (eds.), Loops and Legs in Quantum Field Theory, PoS(LL2012) (2012). Proceedings of the 11th DESY Workshop on Elementary Particle Theory, April 15 - 20, 2012, Wernigerode, Germany. [PoS(LL2012)].

[5] J. Fleischer and T. Riemann, Complete algebraic reduction of one-loop tensor Feynman integrals, Phys. Rev. D83 (2011) 073004, [arXiv: 1009 .4436].

[6] J. Fleischer and T. Riemann, Calculating contracted tensor Feynman integrals, Phys.Lett. B701 (2011) 646-653, [arXiv:1104.4067]. 
[7] A. I. Davydychev, A simple formula for reducing Feynman diagrams to scalar integrals, Phys. Lett. B263 (1991) 107-111. doi:10.1016/0370-2693(91)91715-8.

[8] O. Tarasov, Connection between Feynman integrals having different values of the space-time dimension, Phys.Rev. D54 (1996) 6479-6490, [hep-th/9606018].

[9] J. Fleischer, F. Jegerlehner, and O. Tarasov, Algebraic reduction of one-loop Feynman graph amplitudes, Nucl. Phys. B566 (2000) 423-440, [hep-ph/ 9907327$].$

[10] J. Fleischer, Application of Mellin-Barnes representations to the calculation of massive five-point functions in bhabha scattering, 2007. Talk held at Conference on Frontiers in Perturbative Quantum Field Theory, 14 - 16 June 2007, ZiF, Bielefeld, Germany. Transparencies: fleischer-bielefeld-2007-talk.pdf.

[11] T. Diakonidis, J. Fleischer, J. Gluza, K. Kajda, T. Riemann, and J. Tausk, A complete reduction of one-loop tensor 5- and 6-point integrals, Phys. Rev. D80 (2009) 036003, [arXiv: 0812.2134 ].

[12] J. Fleischer, J. Gluza, M. Gluza, and T. Riemann, OLEC project; C++ and Mathematica packages OLEC v.0.9 available at Silesian Univ., Katowice, webpage http://prac.us.edu.pl/ gluza/olec/.

[13] J. Fleischer, J. Gluza, K. Kajda, T. Riemann, and V. Yundin, Hexagon project; Mathematica package hexagon.m v.1.0 available at Silesian Univ., Katowice, from http://prac.us.edu.pl/ gluza/hexagon/packages/hexagon.m.

[14] J. Gluza, K. Kajda, T. Riemann, and V. Yundin, New results for loop integrals: AMBRE, CSectors, hexagon, PoS ACAT08 (2008) 124, [0902.4830].

[15] J. Gluza and T. Riemann, New results for 5-point functions, eConf C0705302 (2007) LOOP01, [0712.2969].

[16] J. Gluza and T. Riemann, A New treatment of mixed virtual and real IR-singularities, PoS RADCOR2007 (2007) 007, [0801.4228].

[17] T. Diakonidis, J. Fleischer, J. Gluza, K. Kajda, T. Riemann, and J. Tausk, On the tensor reduction of one-loop pentagons and hexagons, Nucl. Phys. Proc. Suppl. 183 (2008) 109-115, [arXiv:0807.2984].

[18] J. Fleischer, T. Riemann, and V. Yundin, PJFry: A C++ package for tensor reduction of one-loop Feynman integrals, . In: [3], preprint DESY 11-25 (2011), http://www-library.desy.de/cgi-bin/showprep.pl?desy11-252.

[19] J. Fleischer, T. Riemann, and V. Yundin, New developments in PJFry, 1210.4095.

[20] V. Yundin, Massive loop corrections for collider physics, $\mathrm{PhD}$ thesis, Humboldt-Universität zu Berlin, 2012, see http://edoc.hu-berlin.de/docviews/abstract.php?id=39163 and urn:nbn:de:kobv:11-100199626.

[21] T. Hahn and M. Perez-Victoria, Automatized one loop calculations in four-dimensions and D-dimensions, Comput.Phys.Commun. 118 (1999) 153-165, [hep-ph/9807565].

[22] T. Hahn, Feynman Diagram Calculations with FeynArts, FormCalc, and LoopTools, PoS ACAT2010 (2010) 078, [1006.2231].

[23] A. van Hameren, Oneloop: For the evaluation of one-loop scalar functions, Comput. Phys. Commun. 182 (2011) 2427-2438, [arXiv: 1007 .4716]. 